\title{
Evaluation of pain relief and satisfaction in women using epidural analgesia in labour
}

\author{
Manisha C. Shembekar ${ }^{1}$, Chaitanya A. Shembekar'2, \\ Shantanu C. Shembekar ${ }^{3}$, Jayshree J. Upadhye ${ }^{4 *}$
}

\begin{abstract}
${ }^{1}$ Department of Anaesthesiology, ${ }^{2}$ Department of Obstetrics and Gynecology Omega Hospital, Nagpur, Maharashtra, India

${ }^{3}$ Department of Obstetrics and Gynecology, Government Medical College, Nagpur, Maharashtra, India

${ }^{4}$ Department of Obstetrics and Gynecology, Narayan Medical College and Hospital, Jamuhar, Bihar, India
\end{abstract}

Received: 07 January 2019

Accepted: 05 February 2019

\section{*Correspondence:}

Dr. Jayshree J. Upadhye,

E-mail: jayshreeupadhye@gmail.com

Copyright: (C) the author(s), publisher and licensee Medip Academy. This is an open-access article distributed under the terms of the Creative Commons Attribution Non-Commercial License, which permits unrestricted non-commercial use, distribution, and reproduction in any medium, provided the original work is properly cited.

\section{ABSTRACT}

Background: Pain during childbirth is the most severe pain women may experience in their lifetime. Epidural analgesia is an effective form of pain relief during labour.

Methods: This retrospective study was carried out at Omega Hospital, Nagpur in 50 women in labour who demanded epidural analgesia for pain relief. It was carried out to determine extent of pain relief and overall satisfaction in women using epidural analgesia.

Results: In present study, out of 50, 28 (56\%) patients delivered by normal vaginal delivery, 14 (28\%) patients delivered by cesarean section, $8(16 \%)$ patients had vacuum delivery while in no patient forceps were applied. In first stage,36 (72\%) patients had zero score (no pain), 13 (26\%) patients had 1-3 score (mild pain) while 1 (2\%) patient had 4-6 score (moderate pain) after epidural analgesia. That means epidural analgesia had very good effect in first stage in terms of pain scoring. In second stage, 13 (26\%) patients had zero score (no pain), 31 (62\%) had 1-3 score (mild pain) while $6(12 \%)$ had 4-6 score (moderate pain) after epidural analgesia. That means epidural analgesia had good effect in second stage in terms of pain scoring. In terms of satisfaction, $11(22 \%)$ patients said it was excellent, $27(54 \%)$ patients said it was good, $8(16 \%)$ patients said it was average while $4(8 \%)$ said it was poor. Apgar score for all babies at 1 min and 5 min was very good. It was $\geq 7$ in all $50(100 \%)$ of newborn babies.

Conclusions: Epidural labor analgesia gives significantly less pain scores and has better maternal satisfaction. It is not associated with increased rate of instrumental vaginal delivery or cesarean delivery. Patients had excellent neonatal outcome.

Keywords: Epidural, Labour analgesia, Pain relief, Satisfaction

\section{INTRODUCTION}

Neuraxial analgesia includes spinal, epidural, and combined spinal-epidural analgesia. Epidural analgesia refers to local anesthetics and adjuvant injected into the epidural space. Spinal anesthesia refers to local anesthetic, with or without adjuvant, injected into the subarachnoid space. Combined spinal-epidural analgesia includes analgesia initiated with an intrathecal injection and then epidural catheter is inserted to provide a route for additional drug in order to maintain analgesia. Here, the spinal component provides rapid analgesia with very little motor block of the lower extremities and then an epidural catheter is placed to ensure that analgesia is available throughout labor. ${ }^{1}$ Neuraxial techniques are the 
gold standard for intrapartum labour analgesia. Multiple randomized controlled trials have demonstrated lower maternal pain scores and higher maternal satisfaction with neuraxial analgesia. Also, the physiological benefits of neuraxial analgesia for the mother and fetus are welldocumented. It improves maternal cardiovascular and pulmonary physiology, and the acid-base status of the fetus. $^{2}$

Epidural analgesia is achieved by placing a 16- or 18gauge catheter through a needle inserted into the epidural space. It is an effective form of pain relief during labour. Local anesthetics and/or opioids are administered into the epidural space on either an intermittent and/or continuous basis throughout labour. The drugs act locally on nerve roots which exit the spinal cord and provide segmental analgesia. The epidural catheter may be utilized to administer higher doses of drugs if needed and also for instrumental or cesarean delivery. The catheter may also be left in situ postpartum to manage pain after birth. ${ }^{3}$

Side effects of epidural analgesia are motor block, nausea, shivering, itching and headache due to accidental dural puncture. Epidural analgesia with low concentration infusions of bupivacaine does not increase the incidence of caesarean section but may increase the incidence of instrumental vaginal delivery and the duration of second stage of labour. ${ }^{3}$

As per 2002 recommendations of the American College of Obstetricians and Gynecologists Task Force on caesarean section, obstetric practitioners should delay the administration of epidural analgesia in nulliparous women until cervical dilatation reaches $4 \mathrm{~cm}$ to $5 \mathrm{~cm}$. Other forms of analgesia be used until that time. ${ }^{4}$

Early initiation is typically defined as with cervical dilatation of less than 4 to $5 \mathrm{~cm}$, and late initiation with cervical dilatation of more than 4 to $5 \mathrm{~cm}$. The American College of Obstetricians and Gynecologists (ACOG) recommended for many years that women delay requesting epidural analgesia, 'when feasible, until the cervix is dilated to $4-5 \mathrm{~cm} .^{5}$

ACOG has recommended that irrespective of epidural analgesia in second-stage, dystocia, the need for instrumental or surgical intervention should not be mandated solely based on second stage duration, especially if progress of labour is satisfactory. ${ }^{6}$ Several studies suggest that a prolonged second stage of labour does not result in adverse maternal or fetal outcomes if fetal status is reassuring, the mother is well hydrated and has adequate analgesia with progress in fetal head descent. ${ }^{7}$ Epidural analgesia is recommended for healthy pregnant women requesting pain relief during labour, depending on a woman's preferences. ${ }^{8}$

Epidural analgesia does not increase the risk of caesarean delivery as compared with systemic analgesia. Initiation of epidural analgesia in the latent phase of labour does not increase the rate of Caesarean delivery or prolong the duration of labour. It can prolong the second stage of labour and increase the rate of instrumental vaginal delivery. ${ }^{9}$ Bupivacaine is superior to lidocaine because of its increased duration of action, reduced incidence of tachyphylaxis, and reduced intensity of lower limb motor block. ${ }^{10}$

The well-known visual analogue scale (VAS) and numeric rating scale (NRS) for assessment of pain intensity agree well and are equally sensitive in assessing acute pain after surgery, and they are both superior to a four-point verbal categorical rating scale (VRS). Its corelation is as follows ${ }^{11}$ :

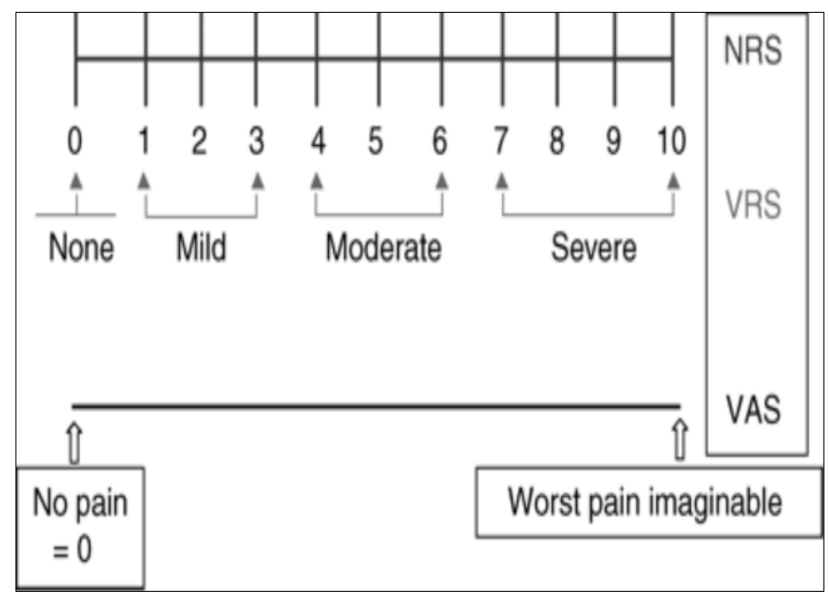

Figure 1: Visual analogue scale (VAS) and numeric rating scale (NRS) for assessment of pain intensity.

The objective of the present study was to evaluate the VAS pain scores and degree of satisfaction in patients receiving epidural analgesia as primary outcome, to evaluate the obstetric and fetal outcomes including risk of caesarean section, instrumental delivery and Apgar score as secondary outcome.

\section{METHODS}

This study was carried out at Omega Hospital, Nagpur in 50 women in labour who demanded epidural analgesia for pain relief during April 2017-March 2018. It was a retrospective study carried out in women in labour who had received epidural analgesia for pain relief in labour with no contraindications to spontaneous delivery and in patients with singleton pregnancies of $>36$ and $<41$ weeks gestation with cephalic presentation

After routine examination and confirming that patient is fit for anesthesia, epidural analgesia was given.

\section{Inclusion criteria}

- Women in labour who demanded epidural analgesia for pain relief with no contraindications to spontaneous delivery. 
- $\quad$ Patients with singleton pregnancies of $>36$ and $<41$ weeks gestation with cephalic presentation.

\section{Exclusion criteria}

- Allergy to opioids, a history of the use of centrallyacting drugs, chronic pain, and psychiatric diseases

- Women younger than 18 year or older than 45 year

- Those who were not willing or could not finish the whole study

- Alcohol- or opioid-dependent patients

- Women with a non-vertex presentation or scheduled induction of labor

- Diagnosed diabetes mellitus and pregnancy-induced hypertension

- Twin gestation and breech presentation.

- Pre-eclampsia, macrosomia, non-reassuring fetal heart rate tracing, abnormal biophysical profile or oxytocin stress test.

After intravenous $500 \mathrm{ml}$ Ringer lactate solution, patients were placed in the flexed lateral position. After raising a midline skin wheal with $1 \%$ lidocaine, the epidural space was identified using loss of resistance to air $(2 \mathrm{ml})$ at the L2-L3 or L3-L4 level, and a multiport epidural catheter was advanced $5 \mathrm{~cm}$ into the epidural space.

Bupivacaine $0.125 \% \quad 10 \mathrm{ml}$ with 2 micrograms $/ \mathrm{ml}$ of Fentanyl was used as the initial dose. Top up dose of $5 \mathrm{ml}$ $0.125 \%$ Bupivacaine with 2 microgram $/ \mathrm{ml}$ of Fentanyl was given on demand, not earlier than 30 minutes of the initial dose.

Pain score (VAS), and pulse, mean arterial pressure, respiratory rate were recorded before epidural, $5 \mathrm{~min}, 15$ min and then every 15 min till 1 hour and then every 30 min until the delivery. Sensory block height was assessed by loss of sensation to pin prick.

Onset of analgesia was defined as duration from injection of first initial epidural bolus dose till VAS <3. Duration of analgesia of initial bolus dose was defined as time of administration of study drug until the time of demand of top-up for the first time.

The time taken by the women to request for subsequent top-up dose was recorded. Labor was managed according to obstetric protocols and mode of delivery (normal/instrumental delivery/caesarean delivery) was noted. Injection to delivery interval was defined as the time from administration of first initial epidural dose until the delivery. Fetal heart rate was monitored by a cardiotocograph. Neonatal assessment was done by Apgar score at 1 and $5 \mathrm{~min}$.

Quality of analgesia was assessed as follows-

- Grade 0 - Poor

- Grade 1 - Average
- Grade 2 - Good,

- $\quad$ Grade 3 - Excellent,

- Grade 4-Delivered by cesarean section.

Side-effects including nausea, vomiting, hypotension, hypersensitivity reaction, shivering, fever, drowsiness, pruritus, respiratory depression, retention of urine, and weakness in limbs were noted.

\section{RESULTS}

In present study, duration of first stage was 4-6 hours in $24(48 \%)$ of patients, $6-8$ hours in $12(24 \%)$ of patients while it was <4 hours in $12(24 \%)$ of patients. 2 patients had duration of first stage of $>8$ hours (Table 1 ).

Table 1: Duration of first stage.

\begin{tabular}{|l|l|l|}
\hline Duration of first stage & No. of patients & $\%$ \\
\hline$<4$ hours & 12 & 24 \\
\hline 4-6 hours & 24 & 48 \\
\hline $6-8$ hours & 12 & 24 \\
\hline$>8$ hours & 2 & 4 \\
\hline
\end{tabular}

So, in almost half of the patients, first stage was of less duration. In present study, duration of second stage was $30 \mathrm{~min}-1$ hour in $29(58 \%)$ of patients, it was <30 min in $19(38 \%)$ patients while it was $>1$ hour in $2(4 \%)$ patients (Table 2).

Table 2: Duration of second stage and oxytocin requirement.

\begin{tabular}{|l|l|l|}
\hline Duration of second stage & No. of patients & $\%$ \\
\hline$<30$ min & 19 & 38 \\
\hline 30 min-1 hours & 29 & 58 \\
\hline$>1$ hour & 2 & 4 \\
\hline Oxytocin requirement & & \\
\hline Yes & 33 & 66 \\
\hline No & 17 & 34 \\
\hline
\end{tabular}

$33(66 \%)$ patients required oxytocin for augmentation of labour, while 17 (34\%) patients did not require augmentation of labour by oxytocin.

Table 3: Mode of delivery.

\begin{tabular}{|l|l|l|}
\hline Mode of delivery & No. of patients & $\%$ \\
\hline Normal Vaginal delivery & 28 & 56 \\
\hline Delivery by Vacuum & 8 & 16 \\
\hline Delivery by forceps & 0 & 0 \\
\hline Cesarean section & 14 & 28 \\
\hline
\end{tabular}

So, in more than half of the patients, second stage was of less duration. But more than half of patients required oxytocin for augmentation of labour. In present study, 28 $(56 \%)$ patients delivered by normal vaginal delivery, 14 (28\%) patients delivered by cesarean section, $8(16 \%)$ patients had vacuum delivery while in no patient forceps 
was applied. (Table 3). In present study, in first stage,36 $(72 \%)$ patients had zero score (no pain), $13(26 \%)$ patients had 1-3 score (mild pain) while $1(2 \%)$ patient had 4-6 score (moderate pain) after epidural analgesia. That means epidural analgesia had very good effect in first stage in terms of pain scoring.
In present study, in second stage, $13(26 \%)$ patients had zero score (no pain), 31 (62\%) patients had 1-3 score (mild pain) while $6(12 \%)$ patient had 4-6 score (moderate pain) after epidural analgesia. That means epidural analgesia had good effect in second stage in terms of pain scoring (Table 4).

Table 4: NRS, VRS and VAS after epidural analgesia.

\begin{tabular}{|l|l|l|l|l|}
\hline NRS, VRS and VAS & No. of patients in first stage & $\%$ & No. of patients in second stage & $\%$ \\
\hline 0 (none) & 36 & 72 & 13 & 26 \\
\hline $1-3$ (Mild) & 13 & 26 & 31 & 62 \\
\hline $4-6$ (Moderate) & 01 & 2 & 06 & 12 \\
\hline 7-10 (Severe) & 00 & 0 & 00 & 0 \\
\hline 10 (worst) & 00 & 0 & 00 & 0 \\
\hline
\end{tabular}

However, $31(62 \%)$ patients had pain score of 1-3 in second stage. So, most of the patients delivered vaginally. In present study, in terms of satisfaction for the effect of epidural analgesia, $11(22 \%)$ of patients said it was excellent, $27(54 \%)$ of patients said it was good, $8(16 \%)$ of patients said it was average while $4(8 \%)$ said it was poor (Table 5).

Table 5: Patient satisfaction.

\begin{tabular}{|l|l|l|}
\hline Patient satisfaction & No. of patients & $\%$ \\
\hline Excellent & 11 & 22 \\
\hline Good & 27 & 54 \\
\hline Average & 08 & 16 \\
\hline Poor & 04 & 08 \\
\hline
\end{tabular}

So, patient satisfaction was good in most cases. In present study, Apgar score for all babies at $1 \mathrm{~min}$ and $5 \mathrm{~min}$ was very good. It was $\geq 7$ in all $50(100 \%)$ of new-born babies (Table 6).

Table 6: Apgar scores.

\begin{tabular}{|l|l|l|} 
Apgar scores & $\begin{array}{l}\text { Apgar scores at 1 } \\
\text { min }\end{array}$ & $\begin{array}{l}\text { Apgar scores at 5 } \\
\text { min }\end{array}$ \\
\hline$<7$ & 00 & 00 \\
\hline$\geq 7$ & 50 & 50 \\
\hline
\end{tabular}

So, neonatal outcome was very good in all patients.

\section{DISCUSSION}

In present study, duration of first stage was 4-6 hours in $24(48 \%)$ patients, 6-8 hours in $12(24 \%)$ patients while it was <4 hours in $12(24 \%)$ of patients. 2 patients had duration of first stage of $>8$ hours (Table 1 ).

Contrary to present study, Anim-Somuah M et al in 2005 Cochrane review reported no difference in the duration of the first stage of labour among women receiving epidural analgesia as well as those receiving systemic opioid analgesia or no analgesia. ${ }^{12}$ In present study, duration of second stage was 30 min-1hour in $29(58 \%)$ patients, it was $<30 \mathrm{~min}$ in $19(38 \%)$ patients while it was $>1$ hour in $2(4 \%)$ patients (Table 2$)$.

Contrary to present study, Bogod DG et al found that stage I was significantly longer in the control group (mean 11.1 v. $9.5 \mathrm{~h}(\mathrm{P}<0.05)$ ). Stage II was longer in the infusion group (mean 106 minutes v. 86 minutes), but the difference was not significant $(0.1>\mathrm{P}>0.05){ }^{13}$

Similar to present study, Fyneface-Ogan $\mathrm{S}$ et al found that the mean duration of the first and second stages of labor were significantly shorter in the epidural group when compared with those in the non-epidural group ([P $<0.01]$ and $[\mathrm{P}<0.02]){ }^{14}$

In present study, $28(56 \%)$ patients delivered by normal vaginal delivery, 14 (28\%) patients delivered by cesarean section, $8(16 \%)$ patients had vacuum delivery while in no patient, forceps was applied (Table 3 ).

Contrary to present study, Yancey MK et al found that even after increased use of epidural analgesia, the Caesarean delivery rate in nulliparous women in spontaneous labour was same. $(19.0 \%$ vs $19.4 \%) .{ }^{15}$

Similar to present study, Impey L et al found that, the Caesarean delivery rate remained unchanged (4\% in $1987,5 \%$ in 1992 , and $4 \%$ in 1994; not significant) even after increased rate of epidural analgesia. ${ }^{16}$

In present study, in first stage,36 (72\%) of patients had zero score (no pain), 13 (26\%) of patients had 1-3 score (mild pain), while $1(2 \%)$ patient had 4-6 score (moderate pain) after epidural analgesia. That means epidural analgesia had very good effect in first stage in terms of pain scoring. 
In present study, in second stage, $13(26 \%)$ of patients had zero score (no pain), $31(62 \%)$ of patients had $1-3$ score (mild pain) while 6 (12\%) patient had 4-6 score (moderate pain) after epidural analgesia. That means epidural analgesia had good effect in second stage in terms of pain scoring.

However, satisfaction in terms of pain relief is much more in first stage than in second stage of labour. Similar to present study, Chhetty $\mathrm{YK}$ et al found that after epidural analgesia, majority $(75 \%)$ of parturient achieved VAS <3 significantly earlier in group R2 (0-5 min), then in group $\mathrm{R} 1$ (5-15 min) $\mathrm{P}<0.001$.

After 5 min of epidural bolus injection, VAS score was significantly less in group R2 $(1.63 \pm 2.89)$ than in group $\mathrm{R} 1(5.00 \pm 2.89) \mathrm{P}<0.001{ }^{17}$

Similar to present study, Sheiner E et al found that women receiving epidural analgesia experienced significantly less pain during labor as compared to those receiving pethidine (mean VAS scores 5.05 vs. 9.14, respectively; $\mathrm{p}<0.001)$.

The pain scores 24 hour after labor were significantly lower in patients who receive epidural analgesia (1.69 vs. 2.13 , respectively; $\mathrm{p}<0.001) .{ }^{18}$

In present study, in terms of satisfaction for the effect of epidural analgesia, $11(22 \%)$ of patients said it was excellent, 27 (54\%) of patients said it was good, $8(16 \%)$ of patients said it was average while $4(8 \%)$ said it was poor (Table 5).

Similar to present study, Dickinson JE et al found that maternal satisfaction was significantly higher with epidural analgesia than non-epidural analgesia. Overall satisfaction scores for labour and delivery were high for epidural analgesia. ${ }^{19}$

In present study, Authors found that Apgar score for all babies at $1 \mathrm{~min}$ and $5 \mathrm{~min}$ was very good. It was $\geq 7$ in all $50(100 \%)$ of newborn babies (Table 6). Similar to present study, Leighton BL et al found that analgesic method does not affect fetal oxygenation, neonatal $\mathrm{pH}$, or 5-minute Apgar scores. ${ }^{20}$

\section{CONCLUSION}

Epidural labor analgesia gives significantly less pain scores and has better maternal satisfaction. Epidural analgesia is not associated with increased rates of instrumental vaginal delivery or cesarean delivery. Patients receiving epidural analgesia have excellent neonatal outcome.

\section{Funding: No funding sources}

Conflict of interest: None declared

Ethical approval: The study was approved by the Institutional Ethics Committee

\section{REFERENCES}

1. Silva M, Halpern SH. Epidural analgesia for labor: Current techniques. Local and regional anesthesia. 2010;3:143.

2. Jouppila R, Hollmen A. The effect of segmental epidural analgesia on maternal and foetal acid-base balance, lactate, serum potassium and creatine phosphokinase during labour. Acta Anaesthesiologica Scandinavica. 1976;20(3):259-68.

3. Sng BL, Leong WL, Zeng Y, Siddiqui FJ , Assam PN , Lim Y, Chan ES , Sia AT , Early versus late initiation of epidural analgesia for labour, The Cochrane Database of Systematic Reviews. 2014(10):CD007238.

4. American College of Obstetricians and Gynecologists. Task Force on Cesarean Delivery Rates. Evaluation of cesarean delivery. Washington DC: American College of Obstetricians and Gynecologists. 2000.

5. American College of Obstetricians and Gynecologists. Obstetric analgesia and anesthesia. ACOG Practice bulletin No. 36. Obstet Gynecol. 2002;100(1):177-91.

6. American College of Obstetricians and Gynecologists, Dystocia and augmentation of labor. ACOG Practice Bulletin No. 49. Obstet Gynceol. 2003;102(6):1445-54.

7. Dei-ham RJ, Crowhurst J, Crowther C. The second stage of labour: durational dilemmas. Aus New Z J Obstet Gynaecol. 1991;31(1):31-6.

8. WHO recommendation on epidural analgesia for pain relief during labour.

9. Cambic CR, Wong CA, Labour analgesia and obstetric outcomes, BJA: $\mathrm{Br} \mathrm{J}$ Anaesth. 2010;105(1):i50-60.

10. Albright GA. Cardiac arrest following regional anesthesia with etidocaine or bupivacaine. Anesthesiology: J Am Soc Anesthesiol. 1979;51(4):285-7.

11. Breivik EK, Björnsson GA, Skovlund E. A comparison of pain rating scales by sampling from clinical trial data. Clinic J Pain. 2000;16(1):22-8.

12. Anim-Somuah M, Smyth RM, Jones L. Epidural versus non-epidural or no analgesia in labour. Cochrane database of systematic reviews. 2011(12).

13. Bogod DG, ROSEN M, REES GA, Extradural Infusion Of $0.125 \%$ Bupivacaine to women during labour, Br J Anaesth. 1987;59(3):325-30.

14. Fyneface-Ogan S, Mato CN, Anya SE. Epidural anesthesia: views and outcomes of women in labor in a Nigerian hospital. Ann Afr Med. 2009;8(4):250-6.

15. Yancey MK, Pierce B, Schweitzer D, Daniels D. Observations on labor epidural analgesia and operative delivery rates. Am J Obstet Gynecol. 1999;180(2):353-9.

16. Impey L, MacQuillan K, Robson M. Epidural analgesia need not increase operative delivery rates. American J Obstet Gynecol. 2000;182(2):358-63. 
17. Chhetty YK, Naithani U, Gupta S, Bedi V, Agrawal I, Swain L. Epidural labor analgesia: A comparison of ropivacaine $0.125 \%$ versus $0.2 \%$ with fentanyl. J Obstetric Anaesthes Critical Care. 2013;3(1):16.

18. Sheiner E, Shoham-Vardi I, Sheiner EK, Press F, Hackmon-Ram R, Mazor M, Katz M, A comparison between the effectiveness of epidural analgesia and parenteral pethidine during labor. Arch Gynecol Obstet. 2000;263(3):95-8

19. Dickinson JE, Paech MJ, McDonald SJ, Evans SF, Maternal satisfaction with childbirth and intrapartum analgesia in nulliparous labour. Aust NZJ Obstet Gynaecol. 2003;43(6):463-8.
20. Leighton BL, Halpern SH. The effects of epidural analgesia on labor, maternal, and neonatal outcomes: a systematic review. Am J Obstet Gynecol. 2002;186(5):S69-77.

Cite this article as: Shembekar MC, Shembekar CA, Shembekar SC, Upadhye JJ. Evaluation of pain relief and satisfaction in women using epidural analgesia in labour. Int J Reprod Contracept Obstet Gynecol 2019;8:1016-21. 